\title{
Networks-in-Package; Design, Analysis and Implementation
}

\author{
Gawon Kim, *Kangmin Lee, **Jinhan Kim, **Kicheol Bae, **Choonheung Lee, \\ Hoi-Jun Yoo and Joungho Kim
}

\begin{abstract}
Terahertz Interconnection and Package Laboratory, Department of Electrical Engineering, Korea Advanced Institute of Science and Technology, 373-1 Guseong-dong, Yuseong-gu, Daejeon 305-701, Korea. Lab. homepage) tera.kaist.ac.kr, Tel) +82-42-879-9869. Fax) +82-42-869-8058,

E-mail) gawon4u@eeinfo.kaist.ac.kr, teralab@ee.kaist.ac.kr

* Samsung Electronics Co. Seoul, Korea, **Amkor Technology Korea, Seoul, Korea
\end{abstract}

\begin{abstract}
SiP(System-in-Package) and SoC(System-on-Chip) are familiar to us. In this paper, we firstly define advanced concepts of NoC(Network-on-Chip) and NiP (Network-in-Package). Design and implementation of $\mathrm{NoC}$ are explained and then, $\mathrm{NiP}$ used for $\mathrm{NoC}$ is designed and analyzed regarding of signal integrity and power integrity. The low-power packet-switched NoC with hierarchical star topology is designed and implemented for high-performance SoC platform. An NiP integrating four NoCs is fabricated in a 676-BGA-type package for large and scalable systems and the measured results of the NiP show perfect communications between NoCs.
\end{abstract}

\section{Introduction}

Recently, there have been strong demands for high-density packages which include multiple chips and passive components. A System-in-Package (SiP) (or SoP, that is System-on-Package) technology has been proposed as a way to overcome the density limitation of conventional single-chip package [1]. However, there is no significant difference between SiP and simply connected Multi-Chip-Module (MCM) except for their size or stacking. A concept of Networks-in-Package (NiP) is multi-chip module in a single package with packetswitched communications between Intellectual Properties (IPs) of each chip, as depicted in Fig 1. NiP is an advanced concept of SiP.

Large scale System-on-Chips with huge chip size such as embedded memory logic systems often suffer from their low yield and high cost problems. In this work, a hierarchically star-connected Networks-on-Chip (NoC) refers a designed and implemented using layered protocols and packet-switched networks, and integrated in NiP. For large scale NoC implementations, the power consumption on the network infrastructure should be minimized for reliable transmission of data with low-cost [2]. In this context, we firstly mention used chips, NoCs, and their features. Then, signal integrity and power integrity issues in design and implementation of NiP are shown.

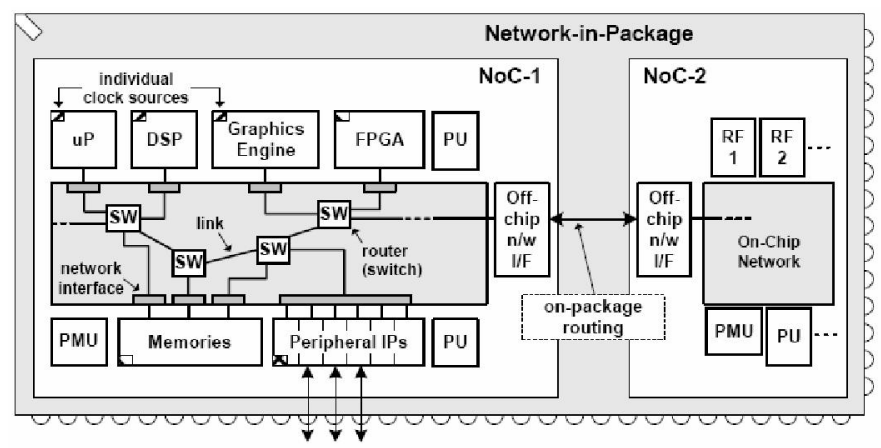

Fig.1. Networks-on-Chip and Networks-in-Package architecture

\section{Design and Implementation of Networks-on-Chip}

An energy-efficient Networks-on-Chip (NoC) is presented for possible applications of high-performance SoC design. It incorporates heterogeneous Intellectual Properties (IPs) such as multiple RISCs and SRAMs, a reconfigurable logic array, an off-chip gateway, and a $1.6 \mathrm{GHz}$ PLL. Its hierarchically-star-connected on-chip 
network provides the integrated IPs, which operate at different clock frequencies, with packet-switched serialcommunication infrastructure. Various low-power techniques such as small-swing signaling, partially activated crossbar, serial link coding, and clock frequency scaling are devised and applied to achieve the power-efficient on-chip communications. The $5 \mathrm{~mm} \times 5 \mathrm{~mm}$ chip containing all above features is fabricated by $0.18 \mu \mathrm{m}$ CMOS process, successfully measured, and demonstrated on a system evaluation board where multimedia applications run. The fabricated chip can provide $11.2 \mathrm{~GB} / \mathrm{s}$ aggregated bandwidth at $1.6 \mathrm{GHz}$ signaling frequency. The chip consumes $160 \mathrm{~mW}$ and the on-chip network dissipates less than $51 \mathrm{~mW}$.
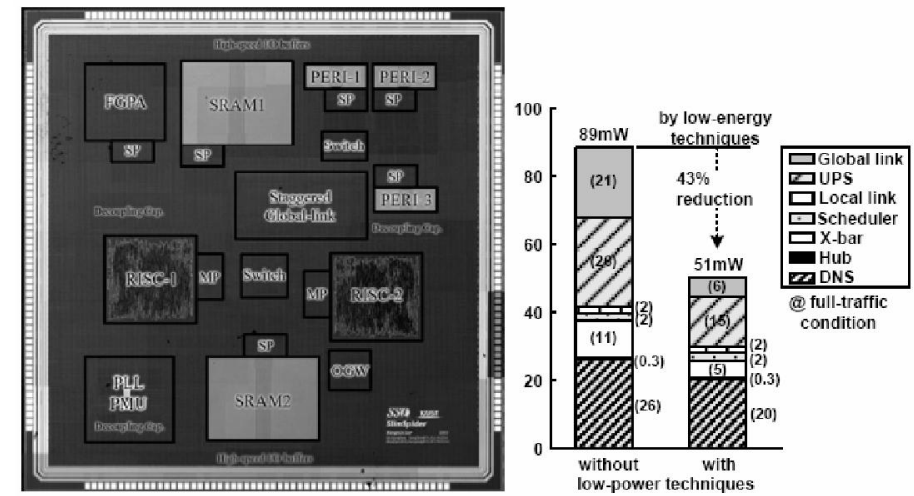

Fig.2. Die photograph and power reduction new techniques

\section{Design and Analysis of Networks-in-Package (NiP) with Pre-Simulation}

The NiP needs four isolated supply voltages: $1.8 \mathrm{~V}$ for digital logic, $1.8 \mathrm{~V}$ for analog circuits, $3.3 \mathrm{~V}$ for $\mathrm{I} / \mathrm{O}$, and sub-0.5V for small-swing links. Each module has different operating frequencies: $50 \mathrm{MHz}$ for peripheral logic, $100 \mathrm{MHz}$ for processors, $800 \mathrm{MHz}$ for scheduler, and $1.6 \mathrm{GHz}$ for on-chip networks.

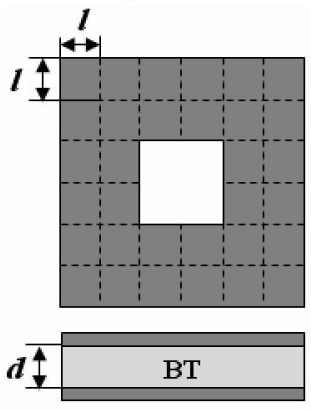

(a) TLM Model of Logic Power

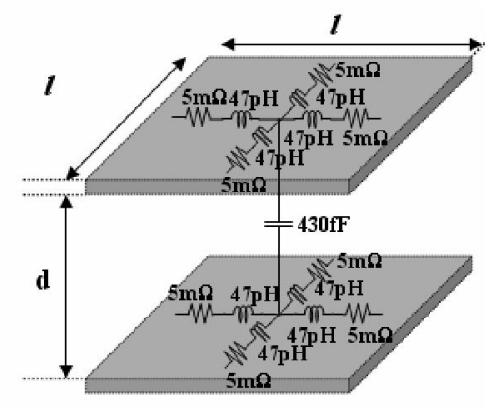

(b) Unit Cell of TLM Model

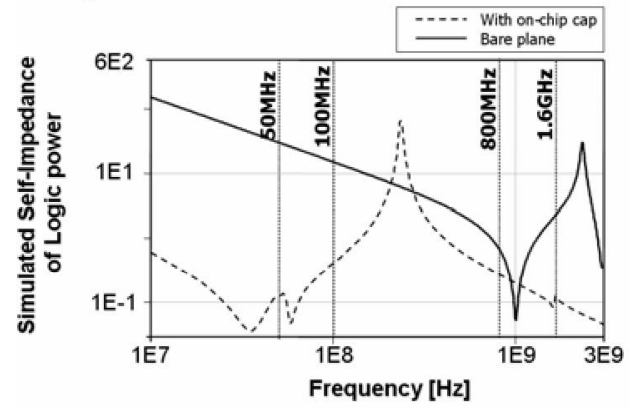

(c) Self-Impedance of Logic Power
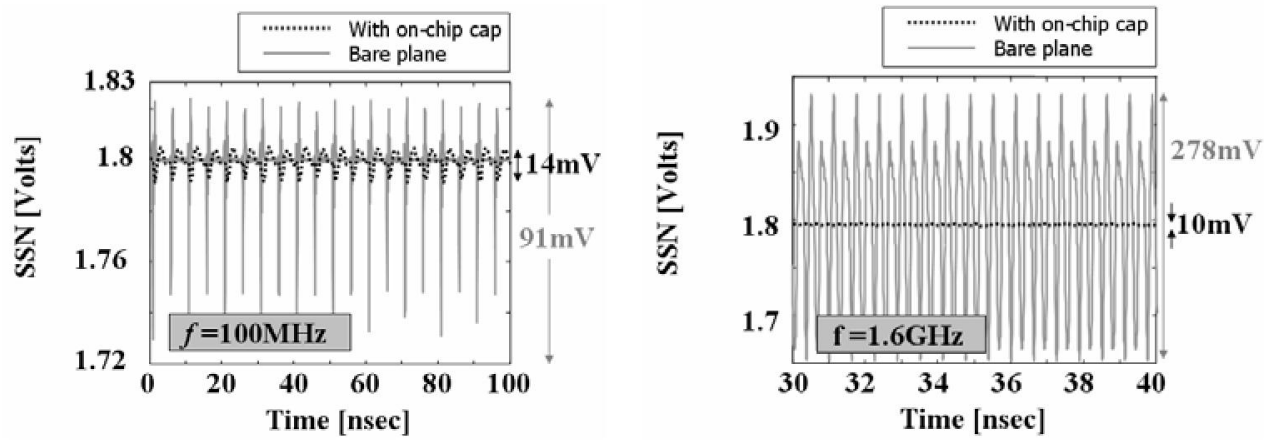

(d) SSN simulations at $100 \mathrm{MHz}$ and $1.6 \mathrm{GHz}$

Fig.3. Logic Power Plane of NiP

One important issue of the $\mathrm{NiP}$ package design is power integrity, i.e. a design of power and ground (P/G) distribution network (PDN). There should be no significant resonance of impedance on the P/G plane of the package at the operating frequency of NoC. In order to analyze the power integrity, we used Balanced 
Transmission Line Matrix (TLM) method and Simultaneous Switching Noise (SSN) analysis [3]. Shapes of power planes to supply three isolated voltages are simulated using TLM method and determined not to have plane-resonances of self-impedances at operating frequencies of the chip, as shown in Fig. 3 (c). We also designed the number of decoupling-capacitors to be used and the values of those for each power voltage at the proper position [4]. As changing shapes of power planes and the number of off-chip decoupling-capacitors, we analyzed induced SSN between power and ground. After inserting the off-chip decoupling-capacitors and onchip decoupling capacitors, the impedance resonance occurs at $272.1 \mathrm{MHz}$, and the SSN voltages are dramatically suppressed at target frequencies, as shown in Fig.4. (d). As a result, self-impedances at the target frequencies become less than $1 \Omega$.

Another issue of the NiP package design is signal integrity, which is reliance on transmission of the highfrequency signals. We designed under some rules considering the signal integrity: separation between the highfrequency signals, e.g. $800 \mathrm{MHz}$ signals or $1.6 \mathrm{GHz}$ signals, the shortest path of high-frequency signals, the maximum number of ground balls for guarantee of return current path, and insertion of ground balls around differential signals to sustain differential mode [5].

\section{Implementation and Measurement of Networks-in-Package (NiP)}

The Networks-in-Package (NiP) system is constructed with four NoCs mounted on a single 676-BGA (Ball Grid Array) package as shown in Fig. 4. The NiP Package has a four-layered PCB, top/ground/power/bottom. The second layer is used as ground (or reference voltage) plane which does not have separation between analog ground and digital ground. Logic power and analog power exist in the third layer, while $\mathrm{I} / \mathrm{O}$ power does in the forth layer. Photographs of implemented $35 \mathrm{~mm} \times 35 \mathrm{~mm}$ NiP package are depicted in Fig.4. (b) and (c).
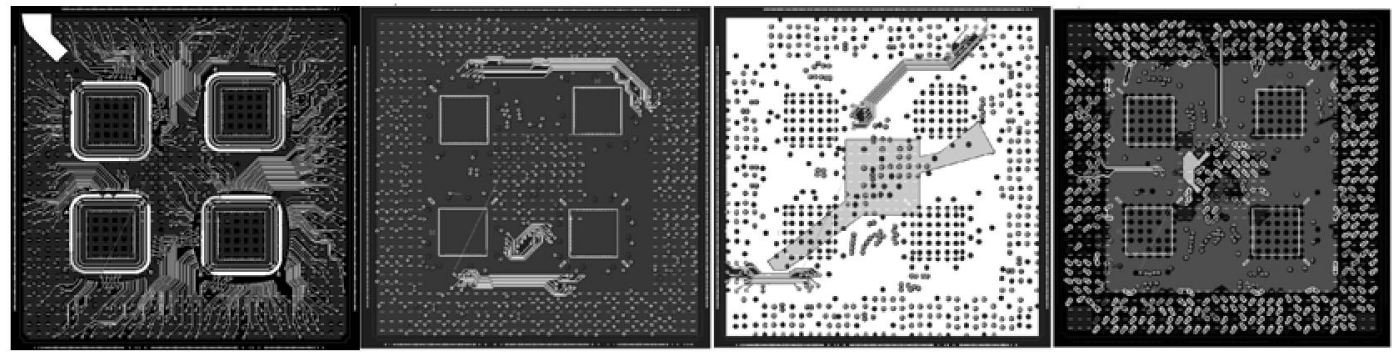

(a) NiP Package Layout (Top/Ground/Power/Bottom)

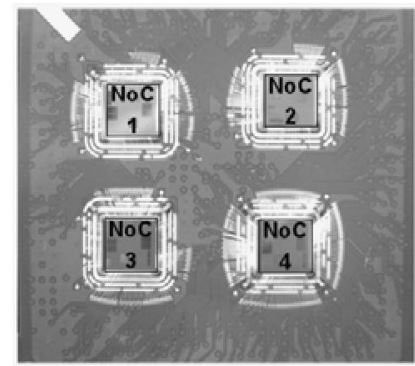

(b) Implemented NiP Package (Before molding)
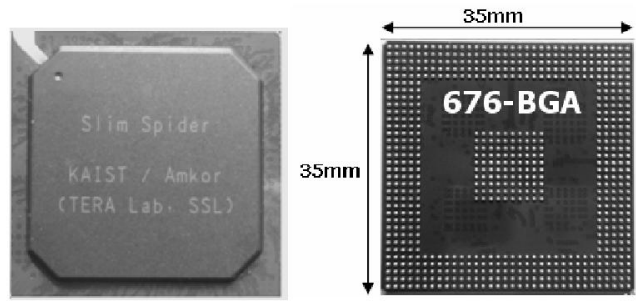

(c) Implemented NiP Package (After molding)

Fig.4. NiP Photograph

Total NiP system is implemented for measurement of operation as described in Fig.5. A NiP test board contains mode-selection switches, frequency-selection switches, high-speed signal pads, reference-clock oscillator, power/ground terminals, and external I/O test pins for operating test.

Measured self-impedance curve of I/O power is plotted in Fig.6 (a). It is noted that the impedance curve has no plane-resonances at operating frequencies of the chip as we intended and it became small impedance

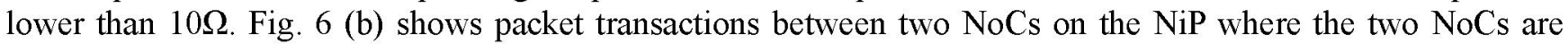
running at different clock frequencies, e.g. $400 \mathrm{MHz}$ and $274 \mathrm{MHz}$. Because the NoC adopts source-synchronous signaling, the $\mathrm{NiP}$ enables plesiochronous communications between processing units running at different clock frequencies in different NoCs. First, NoC-1 commands "Write packet" at NoC-2 and NoC-1 again commands "Read packet at same address" at NoC-2. Then, NoC-2 responses to the "Read command" of NoC-1. 


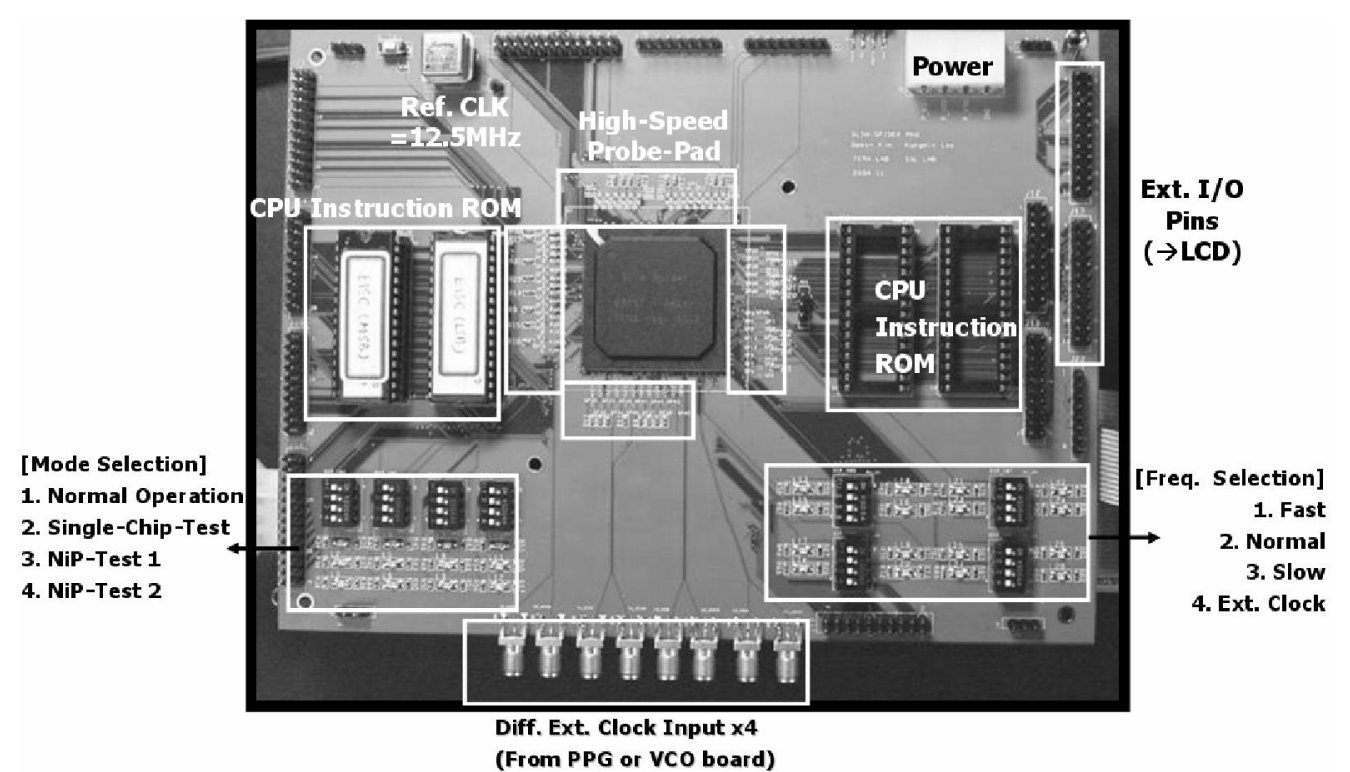

Fig.5. NiP System Implementation

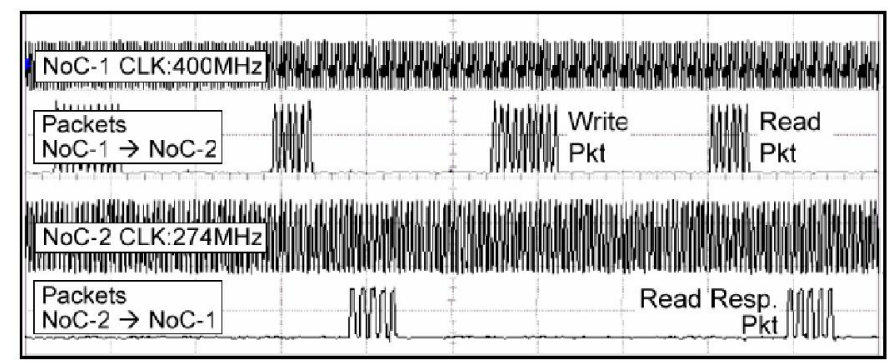

(a) Measured self-Impedance of I/O power (b) Communication signals between NoC-1 and NoC-2 Fig.6. Measurement Results of NiP

\section{Conclusion}

Low-power packet-switched Networks-on-Package (NoC) and Networks-in-Package (NiP) with hierarchical star topology are designed and implemented for high-performance large and scalable system. The NoC contains several processors for chip operation, off-chip gateway for off-chip network interface, and on-chip networks connecting those processing units. Source-synchronous signaling enables plesiochronous communications between processing units in different NoCs running at different clock frequencies. The Networks-in-Package (NiP) system is constructed with four NoCs mounted on a single 676-BGA (Ball Grid Array) package sized $35 \mathrm{~mm}$ by $35 \mathrm{~mm}$. Considering signal integrity and power integrity, separated powers and significant signals of the NiP are designed and analyzed by TLM method and SSN analysis. The implemented NiP shows perfect operations of each NoC and communications between NoCs.

\section{References}

[1] Rao R. Tummala, "SOP: What Is It and Why? A New Microsystem-Integration Technology Paradigm-Moore's Law for System Integration of Miniaturized Convergent Systems of the Next Decade", IEEE Trans. Advanced Packaging, vol. 27, no. 2, 2004, pp. 241-249

[2] Kangmin Lee, Se-Joong Lee, Donghyun Kim, Kwanho Kim, Gawon Kim, Joungho Kim, and Hoi-Jun Yoo, "Networkson-Chip and Networks-in-Package for High-Performance SoC Platforms", IEEE Asian Solid Stated Circuits Conference (ASSCC - Design Contest), Nov. 2005.

[3] Jongbae Park, Hyungsoo Kim, Jun So Park, and Joungho Kim, "Characterization of SSN Coupling to Signal Via in Multi-layer PCBs and Packages", IEEE $17^{\text {th }}$ International Zurich Symposium (EMC-Zurich 2006), pp. 328-331

[4] W. Dally and J. Poulton, "Digital Systems Engineering", Cambridge Univ. Press, 1998

[5] Dong Gun Kam, Heeseok Lee, and Joungho Kim, "Twisted Differential Line Structure on High-Speed Printed Circuit Boards to Reduce Crosstalk and Radiated Emission", IEEE Trans. Advanced Packaging, vol. 27, no. 4, 2004, pp. 590-596 